\title{
Creation of Information Systems Design on the Concept of "Accessible Environment": Designing Systems Orienting Information in Public Places
}

\author{
O.A. Kazachkova \\ MIREA - Russian Technological University, Moscow, Russia
}

\begin{abstract}
This article discusses the basic principles of creating an information system design, using the example of navigation in public places, in order to create a space accessible to all categories of people, taking into account the capabilities of people with disabilities. Identified and analyzed the key tasks of the shopping center navigation system, aimed at ensuring the "accessibility" of public places, and its main components. Issues related to factors influencing the design of the navigation system itself and the design of its components, information carriers, are considered.
\end{abstract}

Keywords: universal design, wayfinding system, direct design, signage system.

\section{Introduction}

The concept of an "accessible environment" implies the formation of a space accessible to all categories of people, taking into account, among other things, the capabilities of people with disabilities. Creating a maximum degree of comfort environment is possible without adaptation and special design and does not preclude the use of assistive devices, if necessary [1].

Creating a universal design of any object, including design regarding material objects, as a system of orienting information of public places, where the carriers of systematized information are often material design objects, involves the development of both the design of the whole object and its constituent parts, taking into account the requirements imposed to the system as a whole $[1,2,3]$. Systems of orienting information allow people to orient themselves in any incoming flow of information, organizing it, systematizing, accompanying by cross-references and other appropriate in one way or another methods and methods.

Actually, a system is understood as any object that is simultaneously considered both as a whole, and as an aggregate of heterogeneous elements united in the interests of achieving its goals [4]. In this case, the navigation system in large shopping centers (TC) is considered as a system of orienting information in public places.

To understand the work of the navigation system, and even more so when it is created, it is necessary to understand the essence of the problems that it solves, as well as the organizational processes in which it is included. Navigation systems of shopping centers are constantly being developed, but as such the scientific description of the fundamentals of their creation is absent, in contrast to the description of navigation systems of museums, educational institutions, hospitals and other objects $[4,5,6,7,8]$.

\section{Main Tasks}

The development of the design of the navigation system of a large shopping center involves, at the first stage, the identification of the tasks to be created by the system being created, and the organizational issues into which it should have been included.

The main tasks that any navigation system is designed to solve are to help the consumer of this system of orienting information. In this case, consumers include:

1) the owners and administration of the shopping center (we put in the first place, as customers of the project, who form their demands and wishes, this is a group of consumers whose goals are often not emphasized);

2) shopping center visitors (the main consumer in need of information provided by this system).

Thus, the tasks of the navigation system of the shopping center from the point of view of two groups of consumers are as follows: 1) ensuring the flow of visitors to all areas of the shopping center, including the outlets of major tenants and representatives of wellknown brands, that is, "anchors";

2) the information system should also have an advertising character, reminding once again of those who are well-known and presenting new brands, stores, etc to the buyer;

3 ) providing a simple and intuitive way of orientation at any point of the complex to all categories of visitors, taking into account who they are, such as profits and what is the purpose of their visit to the center.

\section{Design of the navigation system of the shopping center}

On the one hand, the solution of the above-stated tasks is complicated by the mass flow of people and the overall size of the territory on which to navigate. On the other hand, it can be 
facilitated by dividing the space of a shopping center - as is the case with the Vegas shopping center in Moscow: styling for quarters of different countries of the world: East Bazaar, Ginza, Goldstreet, Fashion Avenue ". The streets are recreated by the interior, stylized architecture of the above places. Thereby offering expressive visual identification suitable for this environment.

In addition, it was necessary to initially plan the location of the main tenants - "anchors" so as to "force" the flows of customers to cover the largest possible areas by moving from one "anchor" to another. Pursuing the same goal, the power zone is usually located at the top level.

Designing a navigation system, solving tasks, is also designed to create a friendly atmosphere, including due to ease of orientation: giving the opportunity to freely navigate in unfamiliar areas, we are less subjecting people to stress, releasing time and energy, creating positive emotions, which increases their purchasing power (in the moral and physical terms).

The design of the navigation system, that is, the orientation system is more than just pointers; it combines signs, signs, "anchors", service departments (wardrobes, toilets, power zones, etc.), walkways, stairs, and elevators into a clear unified system that operates throughout the entire operation of the shopping center.

\section{System approach to the design of information media}

The design of information carriers of navigation - navigators should not contradict the environment in which they are located. Thus, the choice of the main types of navigators and their design involved linking them to the installation environment.

The main information carriers of navigation (navigators) on which wiring is carried out (movement of people's flows in a shopping center), expedient for a given shopping center, include:

1) carriers of maps showing the main destinations, routes, passages, stairs and escalators, elevators and local landmarks (in this case "anchors"), that is, pylons (with a printed map), interactive touchscreens with an electronic navigation system, interactive screens;

2) Indicator navigators - pictograms of toilets, escalators, stairs, power zones, parking areas, etc .;

3) suspended signs with wiring to the necessary for the store objects - "anchors", with service wiring (wardrobe, toilets, food zone, parking, stairs, elevators, etc.), wiring according to the type of goods (children's products, clothing, etc.) ;

3) lift stickers with a description of the levels;

4) navigators of external objects - stairs, parking lots, bus stops, etc .;

5) handouts: shopping center map.

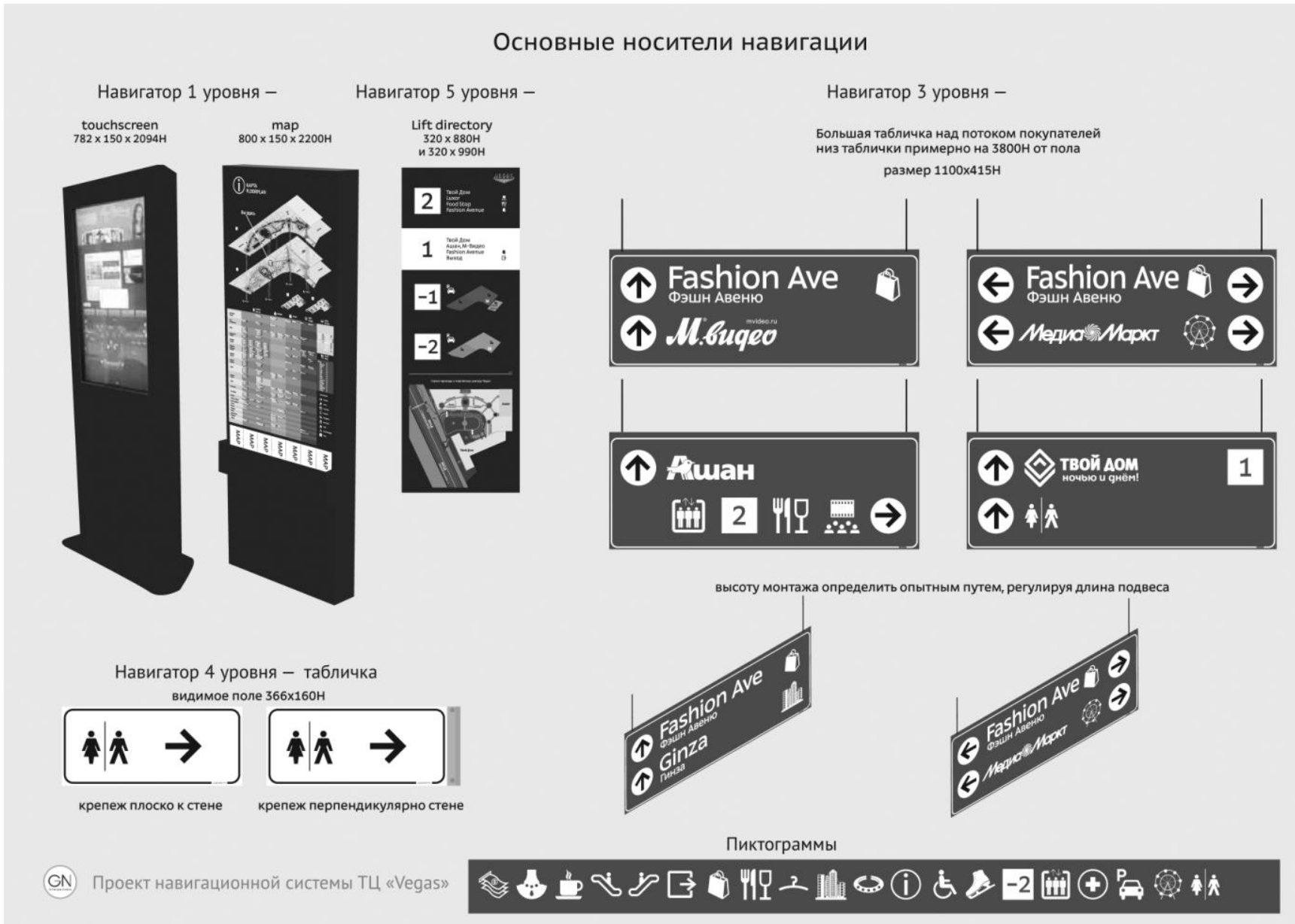

Fig.1: The main information carriers (navigators) in the shopping center "Vegas"

The developed minimum sufficient specification of the navigation system is attached to the map, it includes a list of stores.

Some designers do not bother to solve the issue of installing the system, locating navigators. This approach makes it very difficult for those who install navigators in the mall, given that the number of plates indicating directions to various destinations can exceed several hundred. This issue is recommended to be solved by placing navigators on the master plan, identifying navigators allows digital coding of all navigators.

The design of the navigation system is influenced by the design of the navigators themselves, including the suspension plates. "Design of orientation signs is an art that helps to find the right direction" [5].

A systematic approach to the design of navigators is to solve the 
following questions:

1) Proportionality - the scale of navigators in the environment, fitting: placement frequency, font size, assessment of font readability (choice of future font, its size);

2) Font design - the presence of optical hooks, contrast. The main criteria for choosing a font are the following: whether the image of the company or the project itself is suitable, compatibility or neutrality with respect to the architecture of the object is simple or too florid; , legibility and readable at a distance. The font is estimated visually, in the environment, at different distances and at different angles - the real situation is simulated;

3) Typographic system, which includes a modular grid and font dimensions. Two problems are solved: the scale of the medium in the medium and the proportionality of the information on this medium (how many inscriptions, what, in what type);

4) Design of the visual style pictograms and arrows;

5) Color system - color contrast and compatibility, selection of colors by spot (color systems) fans, transfer from one system to another;

6) The coding system is created and introduced at the beginning of the project, both in the master plan and in navigators, it includes the system of symbols, the system of color coding, etc .;

7) Content of navigators - the content of navigators is created by coding, symbols, naming, color, communication indexes.

In the process of developing a navigation system, it is necessary to remember that the goal of any design project is to make the world better - more beautiful, more accessible, more convenient for a person, the life of people is more interesting, to encourage creation "[1].

\section{Conclusion}

In large shopping centers, there are difficulties in targeting a mass of people: people visiting this center for the first time, or rarely visiting large outlets, people with children, people with disabilities and their accompanying people, including the ones with massive purchases. The main task in all the above cases is to ensure the distribution of flows of people throughout the shopping center, bringing to them the necessary information for the management of the shopping center, creating an "accessible environment" for comfortable movement and shopping, which is in the interests of both buyers and sellers. Architectural solutions, consisting in the availability and thoughtful arrangement of travelators and elevators, are not sufficient. One of the fundamentals is to provide ease of orientation and movement, that is, information carriers should inform their location, as well as other vital objects.

\section{References}

[1] Kazachkova OA, Kumanin V.I. The concept of "design" at the present stage of human development / Informatics and technology: Interuniversity collection. - M .: MGUPI, 2009.

[2] Kazachkova, O.A. Zyabneva, O.A. Mamedova, I.Y. Kulishova E.A. 3D technologies of jewelry with elements of complicated design. International Journal of Engineering and Technology (UAE). - Volume 7, Issue 3, 2018, Pages 155-157.

[3] Sokolova M.L. Modern problems of the direction "Technology of artistic processing of materials." // Russian technological journal. 2017. - Vol. 5, No. 1 (15) - p. 50-56.

[4] William S. Davis, David C. Yen The Information System Consultant's Handbook. Systems Analysis and Design. - CRC Press, 1998. 800 p.

[5] Craig M. Berger. Road signs: Graphic navigation design. - RIP holding, Moscow, 2005. $176 \mathrm{p}$

[6] Andreas Uebele Signage Systems \& Information Graphics: A Professional Sourcebook. - Thames \& Hudson Ltd, London, 2007. $336 \mathrm{p}$.

[7] WalkThis Way: Signe Graphics Now. - Collins Design and maomao publications, New York, 2010. 255 p

[8] The most guiding // Journal [AS], "Offset printing house No. 21",
Moscow, 2007, No. 4 (44). Pp. 4-5, 107. 